\title{
Histopathological and Serological Findings of Lung, Liver and Heart in a Patient with Corona Virus Disease-2019 (COVID -19): A Case Report
}

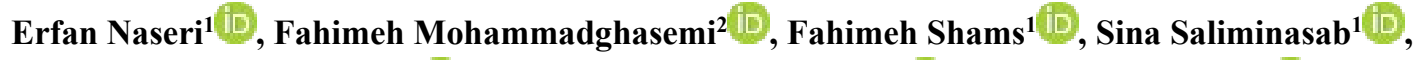 \\ Paridokht Karimian $^{3}$ (D), Morteza Rahbar Taramsari ${ }^{4}$ (D) Siroos Kordrostami $^{5}$ (D), \\ Ali Alavi Foumani ${ }^{6}$, Hossein Hemmati $^{7}$, Seyed Amineh Hojati ${ }^{8}$ (D), \\ Pirouz Samidoust $^{9}$ (D), Masoumeh Faghani ${ }^{*}$ (iD \\ 1. Student Research Committee, School of Medicine, Guilan University of Medical Sciences, Rasht, Iran \\ 2. Cellular \& Molecular Research Center, Guilan University of Medical Sciences, Rasht, Iran \\ 3. Dept. of Pathology, School of Medicine, Guilan University of Medical Sciences, Rasht, Iran \\ 4. Dept. of Forensic Medicine, School of Medicine, Guilan University of Medical Sciences. Rasht, Iran \\ 5. Razi Hospital, School of Medicine, Guilan University of Medical Sciences, Rasht, Iran \\ 6. Inflammatory Lung Diseases Research Center, Dept. of Internal Medicine, Razi Hospital, School of Medicine, Guilan University \\ of Medical Sciences, Rasht, Iran. \\ 7. Dept. of General Surgery, Division of Vascular Surgery, Guilan University of Medical Sciences, Rasht, Iran \\ 8. Gastrointestinal and Liver Diseases Research Center (GLDRC), Razi Hospital, Guilan University of Medical Sciences, Rasht, Iran \\ 9. Dept. of General Surgery, School of Medicine, Guilan University of Medical Sciences, Rasht, Iran
}

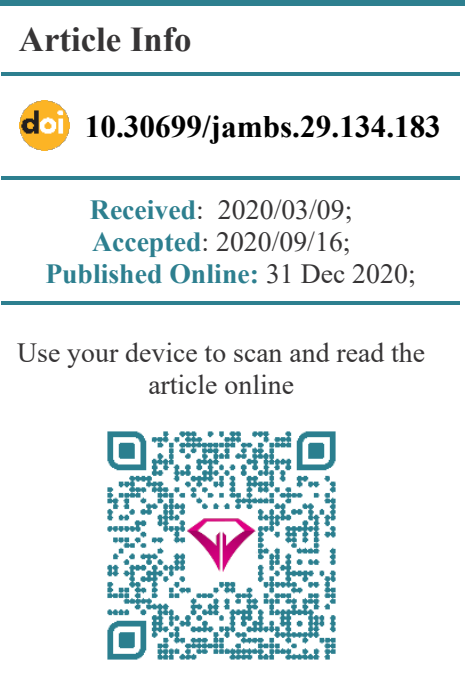

\section{ABSTRACT}

This study aimed to report histopathological features and serological outputs of the lung, heart and liver in a patient suffered from Coronavirus disease-2019 (COVID19). A woman was admitted to the Razi Hospital, Rasht city, Iran with the symptoms of cough, dyspnea, fever and myalgia. She had also Parkinson's disease (PD); she had no history of respiratory, cardiovascular, renal and gastrointestinal diseases and alcohol consumption. COVID-19 infection was proved by the Real time-PCR (RTqPCR) test. The required specimen was obtained from nasopharyngeal swab; however, lung radiologic findings revealed atypical signs of COVID-19. The patient expired after 8 days of admission. After death, needle biopsy was performed for histopathologic evaluation of the heart, lung and liver tissues. The RT-qPCR was performed to evaluate the presence of Coronavirus in the tissues. The pathological biopsies showed advanced alveolar damages in the lung, periportal inflammation in the liver, spread steatosis in the hepatocytes and moderate myocarditis in the heart. However, the RT-qPCR test was negative for these tissues, but alterations in the above-mentioned areas may be due to the secondary side effects of COVID-19 infection or pharmacological treatments.

Keywords: COVID-19, Heart, Liver, Lung, Histopathology

Corresponding Information:

Dr. Masoumeh Faghani, Cellular \& Molecular Research Center, Guilan University of Medical Sciences, Rasht, Iran E-mail: mfaghani2000@gmail.com

\section{Introduction}

The disseminated Coronavirus disease-2019 (COVID19) has almost affected the whole world in the recent months (1). The disease symptoms are variable from mild to severe. It was reported that different body organs are involved by infection, especially lungs and heart (2). It is known that the elderly and people suffering from chronic diseases are more vulnerable to COVID-19 infection (3). There is high similarity in the clinical manifestations of COVID-19 and severe acute respiratory syndrome Coronavirus 2 (SARS-CoV-2); both enter into the tissues through a special receptor called angiotensin-converting enzyme 2 (ACE-2). This receptor is expressed mainly in the respiratory tract and also in various tissues such as kidney, gastrointestinal tract, liver, testis and endothelial cells (4). Therefore, it attacks to numerous tissues. In $23 \%$ of the patients, COVID-19 was associated with the cardiac injury (5). Abnormal liver function (57.8\%) is also observed in the patients (6). In this case report, we described a COVID-19 patient, who died in the hospital after eight days of admission. 


\section{Case Report}

The case study was a 64 year-old woman, who developed left limb bradykinesia in 2005, and was subsequently diagnosed with Parkinson's disease (PD). Later, she had bilateral tremor, rigidity and pain in both upper and lower limbs (left $>$ right). She felt pain; complaining of rising from bed and turning back. The following medications were prescribed: Norstar (Levodopa-B) $250 \mathrm{mg}$ BD, Tranqopine $25 \mathrm{mg}$ BD, Clomipramine $10 \mathrm{mg}$ nightly and Clonazepam $2 \mathrm{mg}$ nightly. She had a background of smoking but quit more than five years ago. She had no history of respiratory, cardiovascular, renal and gastrointestinal diseases and alcohol consumption.

On the date of April $6^{\text {th }}, 2020$, she had some symptoms such as cough, dyspnea, fever and myalgia. Her PD symptoms worsened after fever. Concurrent occurrence of COVID-19 and fever, the patient had mild bilateral ptosis (left >right) that worsened later. General and motors' symptoms became more severe.

She referred to Razi Hospital. The COVID-19 real time PCR (RT-qPCR) test was performed for the nasopharyngeal specimen. The vital signs were reported as follows; respiratory rate $(\mathrm{RR})=28 / \mathrm{min}$, heart rate $=130 / \mathrm{min}$, temperature $=39^{\circ} \mathrm{C}$ and blood pressure $(B P)=140 / 100 \mathrm{mmHg}$. She was transferred to the intensive care unit (ICU). Noninvasive ventilation (with mask $10 \mathrm{Lit} / \mathrm{min}$ ) was applied due to hypoxia and tachypnea.
She seemed ill and pale. The examination findings were as follows; $R R=38 / \mathrm{min}, \mathrm{HR}=136 / \mathrm{min}, \mathrm{BT}=39 / 5 \mathrm{C}$, $\mathrm{BP}=110 / 60 \mathrm{mmHg}$. The arterial blood gas $(\mathrm{ABG})$ analysis showed the following results; $\mathrm{pH}=7.26$, $\mathrm{PCO} 2=59 \mathrm{mmHg}, \quad \mathrm{PO} 2=55.6 \mathrm{mmHg}, \mathrm{HCO} 3=26.2$ $\mathrm{mEq} / \mathrm{L}, \mathrm{O} 2 \mathrm{sat}=83.3 \%, \mathrm{Be}=-1.9 \mathrm{mmol} / \mathrm{L}$ and $\mathrm{BB}=46$ $\mathrm{mmol} / \mathrm{L}$.

Some biochemical laboratory tests, chest X-ray, computed tomography (CT) and brain MRI were requested by physician (Table 1 ). Lung $\mathrm{CT}$ scan revealed bilateral multiple ground glass centrilobular nodules, predominantly in the posterior segment of upper lobes, upper segments of lower lobes and right middle lobe. Mild bronchial wall thickening and dilatation were also noted. No evidence of peripheral ground glass was observed in favor of typical COVID-19. Regarding the patient history and atypical COVID-19, aspiration pneumonia was considered as differential diagnosis.

The heart size was normal (Figure 1). The COVID-19 was suspected according to the chest X-ray. Therefore, Lopinavir, Ritonavir (Kaletra), Vancomycin and Meropenem were prescribed for the patient.

For controlling the PD signs, Levodopa was prescribed. The ECG results showed mild mitral valve and tricuspid valve regurgitation with an ejection fraction (EF) ratio of $55-60 \%$ (other issues were not notable). The cardiac enzymes were not checked, because there was no suspicion of cardiac involvement in the patient.

Table 1. Laboratory results of the patient during hospitalization

\begin{tabular}{|c|c|c|c|c|c|c|c|c|c|}
\hline Variable & Day1 & Day 2 & Day 3 & Day 4 & Day 5 & Day 6 & Day 7 & Day 8 & Normal range \\
\hline WBCs, hpf (U/A) & 19 & N/A & N/A & N/A & 26.4 & 29.9 & N/A & 33.4 & $4-11$ \\
\hline RBCs, hpf (U/A) & 4.02 & N/A & 3.34 & N/A & 3.17 & 4.04 & N/A & 3.89 & $4.5-5.1$ \\
\hline Hemoglobin,gr/dL & 9.4 & 9.6 & 7.9 & 8.7 & 6.4 & 10.3 & 8 & 6.3 & $12.3-15.3$ \\
\hline HCT & 31 & 29.9 & 25.5 & 27.2 & N/A & N/A & N/A & N/A & $35.9-44.6$ \\
\hline PLT & 372 & 362 & 377 & N/A & 263 & 169 & 212 & 204 & $150-450$ \\
\hline CRP, mg/L & +1 & +1 & N/A & N/A & N/A & +2 & N/A & N/A & Negative $:<6$ \\
\hline Creatinine, $\mathrm{mg} / \mathrm{dL}$ & 0.6 & 0.6 & 0.6 & 0.6 & 0.6 & 0.6 & 0.75 & 0.97 & $0.6-1.2$ \\
\hline BUN, mg/dL & 20 & 20 & 19 & 17 & 16 & 16 & 24 & 30 & $13-43$ \\
\hline $\mathrm{Na}, \mathrm{mEq} / \mathrm{L}$ & 134 & 137 & 140 & 143 & 140 & 138 & 172 & 141 & $135-145$ \\
\hline $\mathbf{K}, \mathbf{m E q} / \mathbf{L}$ & 4.1 & 3.8 & 3.9 & 3.6 & 3.5 & N/A & 4.2 & 4.9 & $3.5-5.3$ \\
\hline $\mathbf{M g}, \mathbf{m E q} / \mathrm{L}$ & 1.7 & 1.7 & 2.2 & 3.8 & 2.1 & 1.7 & N/A & 2.3 & $1.53-2.5$ \\
\hline $\mathrm{Ca}, \mathrm{mEq} / \mathrm{L}$ & 9.2 & N/A & N/A & N/A & 9.2 & N/A & 9.7 & N/A & $8.6-10.3$ \\
\hline ALT (SGPT). U/L & 17 & 15 & N/A & 16 & 12 & N/A & 15 & N/A & $<40$ \\
\hline AST (SGOT), U/L & 12 & 7 & N/A & 20 & 17 & 7.6 & N/A & N/A & $<34$ \\
\hline
\end{tabular}




\begin{tabular}{ccccccccccc}
\hline Variable & Day1 & Day 2 & Day 3 & Day 4 & Day $\mathbf{5}$ & Day 6 & Day 7 & Day 8 & Normal range \\
\hline ALP & N/A & 318 & N/A & 276 & 285 & 275 & N/A & N/A & $64-306$ \\
\hline LDH, U/L & 697 & 549 & N/A & N/A & N/A & 987 & N/A & N/A & $230-480$ \\
\hline Blood Sugar (BS) & 119 & 139 & 168 & 137 & 170 & 150 & 152 & N/A & $<200$ \\
\hline Albumin, g/L & 2.8 & 2.2 & N/A & 2.4 & N/A & 3.1 & N/A & N/A & $3.5-5.5$ \\
\hline PT, seconds & 12.5 & 12 & 12 & 12.5 & 12 & 12 & 12 & 12 & $11-13.5 \mathrm{sec}$ \\
\hline PTT, seconds & 37 & 18 & 34 & 35 & 65 & 39 & 64 & 60 & $24-40 \mathrm{sec}$ \\
\hline
\end{tabular}

CRP: C-reactive protein; AST: Aspartate aminotransferase; ALT: Alanine aminotransferase, ALP: Alkaline phosphatase; LDH: Lactate dehydrogenase, PT: Prothrombin time; PTT: Partial thromboplastin time

I
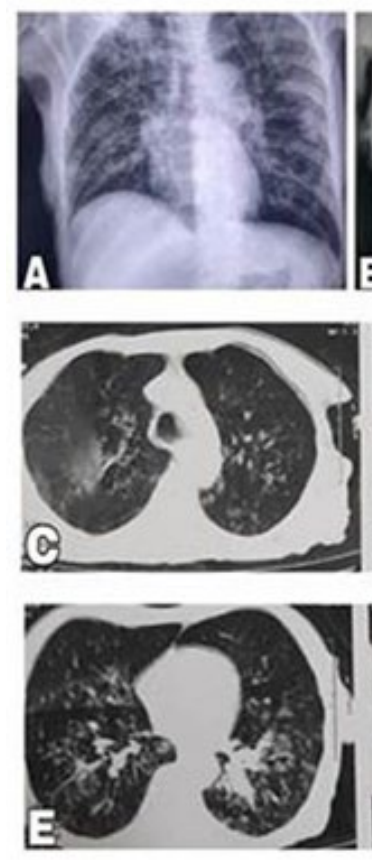
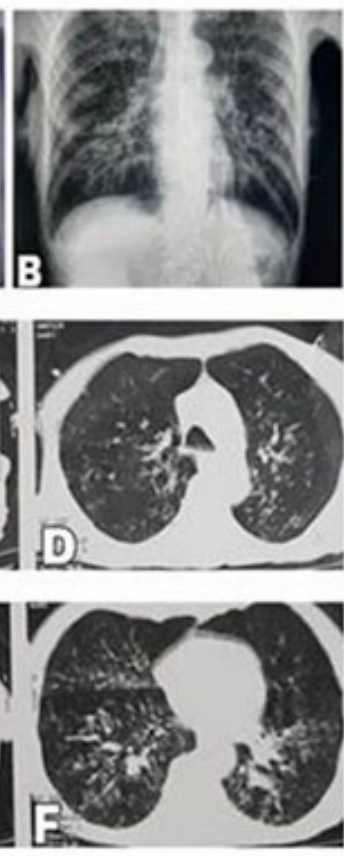

II
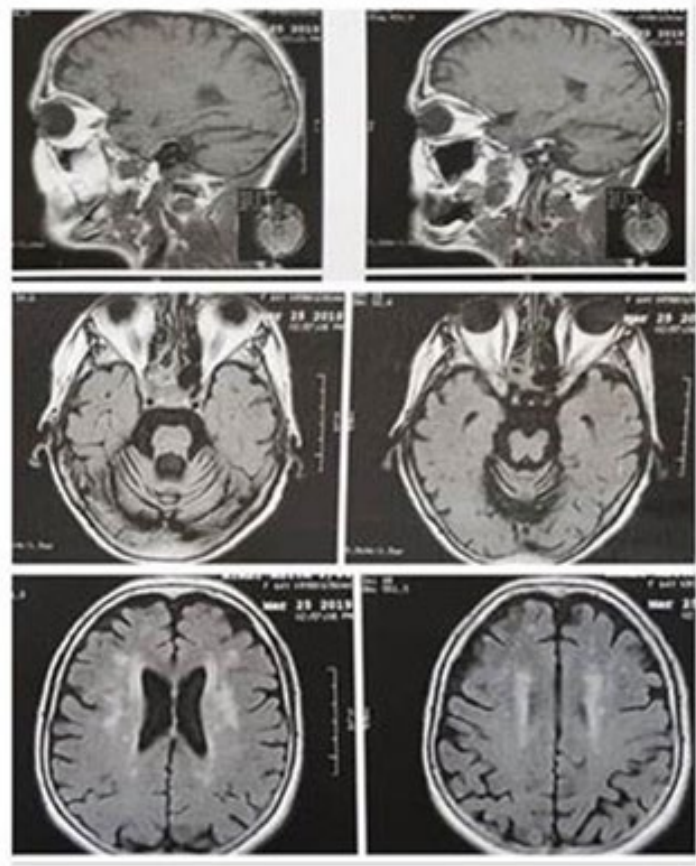

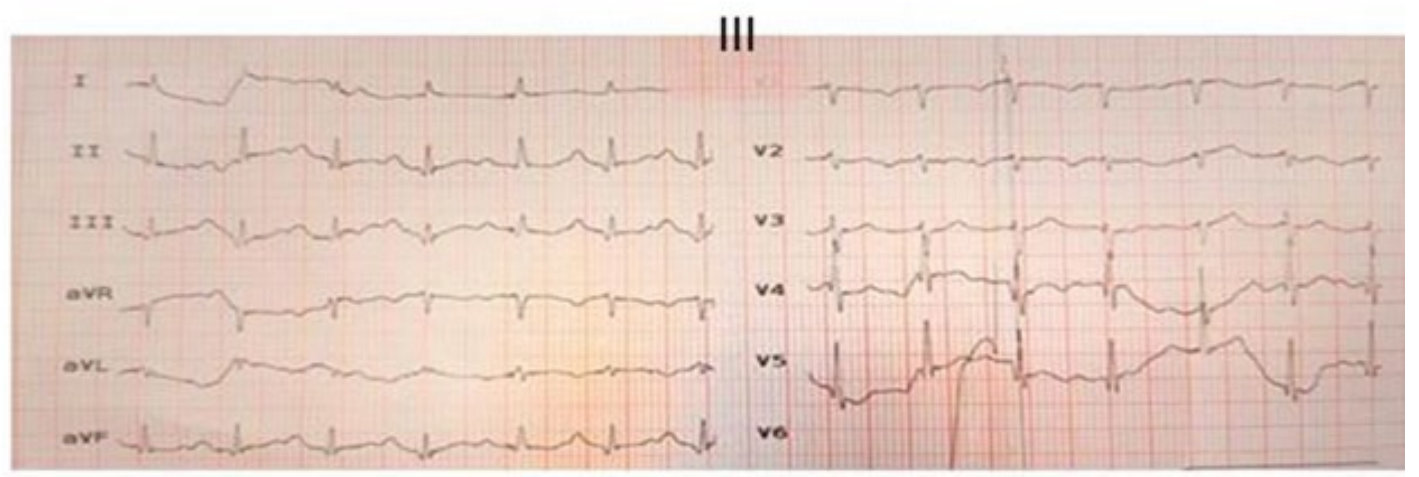

Figure 1. I: Chest X-ray. A: before admission. B: during the admission time which showed faint patchy opacities in the right side and bilateral diffuse hyperinflation. C, D, E and F: The chest axial CT obtained by the time of admission; it revealed bilateral multiple ground-glass centrilobular-nodules in the lungs predominantly in posterior segment of the upper lobes, upper segments of the lower lobes and the right middle lobe. Mild bronchial wall thickening and dilatation were also noted. No evidence of peripheral ground glass in favor of typical COVID-19 was observed. II: Brain MRI. There was multiple small abnormal signal lesions in the brain white matter of periventricular space and sub-cortical of both hemispheres and also basal ganglia; cited alterations were due to small vessel ischemic disease and encephalopathy. Also, senile dilatation of CSF spaces and senile atrophy were seen. III: Electrocardiogram (ECG) showed occasional premature atrial contractions (PAC) and prolonged QT-interval accompanied with first-degree atrioventricular block. 
On day 6 , the patient consciousness reduced. Vital signs and $\mathrm{ABG}$ test assessment were as below: $\mathrm{RR}=22$, $\mathrm{pH}=7.33, \quad \mathrm{PCO} 2=49.3 \mathrm{mmHg}, \quad \mathrm{PO} 2=45.7 \mathrm{mmHg}$, $\mathrm{HCO} 3=26 \mathrm{mEq} / \mathrm{L}$, and $\mathrm{O} 2 \mathrm{sat}=78.3 \%$ (under mask ventilation of $10 \mathrm{Lit} / \mathrm{min}), B e=-0.4 \mathrm{mmol} / \mathrm{L}$ and $\mathrm{BB}=47.5 \mathrm{mmol} / \mathrm{L}$. Finally, she was intubated and underwent mechanical ventilation (mode $\mathrm{A} / \mathrm{C}, \mathrm{FIO} 2$ : 80\%, rate 18/min, VT: $480 \mathrm{ml}$, and PEEP: $10 \mathrm{cmH} 2$ ).At midnight on the last day, BP decreased to $91 / 49 \mathrm{mmHg}$; therefore, one-liter normal saline $0.9 \%$ was injected. BP reduced again, then $10 \mathrm{micro} / \mathrm{min}$ norepinephrine was injected, however, BP did not elevate (BP: 7.1/39 $\mathrm{mmHg}$ ). Additionally, the heart rhythm changed from normal sinus rhythm (NSR) to atrial fibrillation (AF). The patient experienced severe hypotension, and acute respiratory distress syndrome (ARDS) developed. The O2 saturation did not reach higher than 70\% (BP: $65 / 30$ $\mathrm{mmHg}$ ). She did not respond to the cardiopulmonary resuscitation. Finally, she passed away with septic shock diagnosis. A signed consent was obtained from the patient's family for the biopsy.

\section{Histopathology}

Tissue sampling was done from lung, heart and kidney by a non-automatic coaxial biopsy needle $(16 * 20 \mathrm{G})$. The tissues specimens were sent to the pathology lab for histopathologic evaluations. The tissue samples were kept in $10 \%$ formalin solution and embedded by paraffin and stained by Haematoxylin-Eosin (H\&E). The prepared slides were observed via a light microscope. Other parts of the tissues were sent to the virology lab for the COVID-19 detection by RT-qPCR.

\section{Real-time PCR Assay for SARS-COV-2 in Tissues}

Liferiver RNA isolation kit (Belgium) was used for the total RNA extraction. Based on the manufacturer's protocol, total RNA was extracted and RT-qPCR assay was conducted (Catalog number: 8.0131901X024E, Version.B1.0, China). Open reading frame 1ab, Nucleocapsid protein $\mathrm{N}$ and $\mathrm{E}$ genes were amplified via RT-qPCR.

\section{Histopathology Results}

Lung tissue needle biopsy showed increased thickness of the alveolar wall, fibroblastic ball and mild fibrosis in the interstitial tissue. Moreover, pneumocytes type II hyperplasia with giant cell formation in the lung alveolar wall was associated with inflammatory cells in the alveolar spaces; probably, mentioned alterations were due to compensatory response to produce more surfactant. These changes were nonspecific and corresponding to the organizing phase of pneumonia (Figure 2.I).

Histopathological observations of the heart tissue revealed mild inflammatory cell infiltration in both endomysium and perimysium with edema; this finding was consistent with mild myocarditis (Figure 2.II).

Wide sinusoids and congestion were observed in the parenchyma of the liver tissue. Micro-vesicular steatosis in periportal hepatocytes and hepatocyte size heterogeneity were observed. There was also mononuclear infiltration in the portal area. These findings were considered as nonspecific changes (Figure 2.III).

\section{Rt-PCR Results}

The test result was negative in terms of virus presence in the lung, liver and heart tissues.
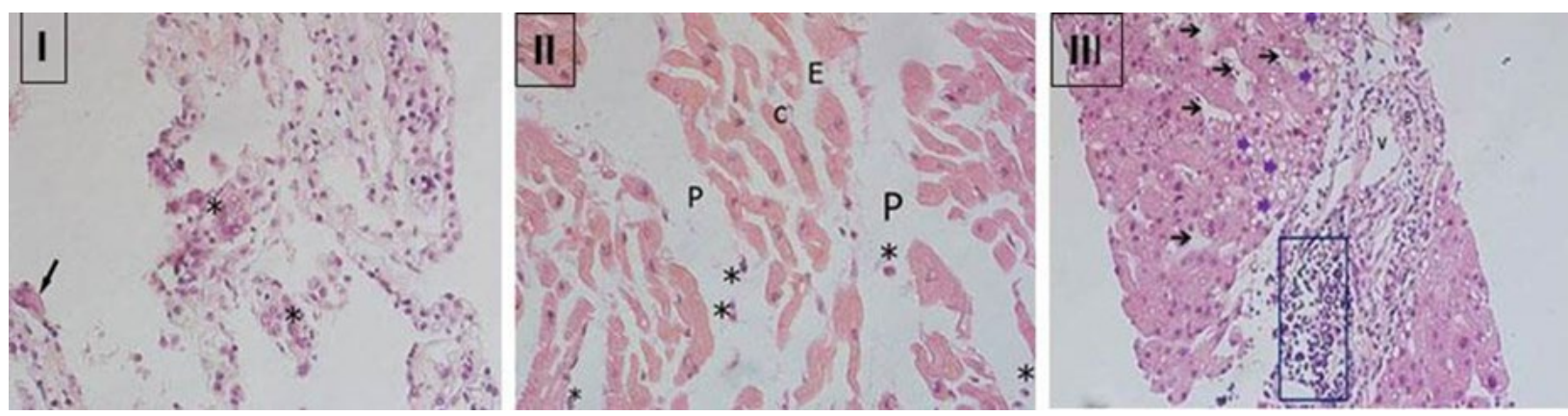

Figure 2. I: Biopsy specimen of the lung tissue. The arrow shows a giant cell. *: Pneumocyte hyperplasia of type 2 with giant cell formation. II: autopsy specimen of the heart tissue. E: endomysium, C: cardiomyocyte, P: perimysium, *: inflammatory cells. III: Biopsy specimen of the liver tissue. Purple arrows: steatosis in hepatocytes. Black arrows: sinusoids, V: portal vein, B: bile duct. The blue rectangle shows inflammation around the portal area. Hematoxylin and eosin 400X.

\section{Discussion}

This study reported clinical and histopathological findings of lung, heart and liver of a COVID19 infected patient. No evidence of peripheral ground glass was noted in the lung CT, in favor of typical COVID-19, which is not special for COVID-19 diagnosis. However, it is usually found in aspiration pneumonia 
(7). Typical sign of COVID-19 is bilateral subpleural patches of ground glass opacity $(7,8)$. Our pathological assays showed alterations which represented organized pneumonia in the lung tissue. These alterations lead to respiratory difficulty. The COVID-19 causes acute pneumonia and its clinical symptoms, which are similar to SARS-CoV and MERS-CoV (9). It should be noted that combination of PCR test, radiologic and CT findings and clinical data is the gold standard manner to recognize COVID-19 infection (8). The studied patient suffered from PD for years; therefore, it seems that she had aspiration pneumonia with a positive nasopharyngeal RT-qPCR for the COVID-19 infection.

The ECG and histopathological findings showed developed mild myocarditis. It also should be noted that she had no previous history of cardiovascular diseases. Cardiac involvement is usually associated with the COVID-19, and it does not have a good prognosis. The possible mechanisms of cardiac injury in the COVID-19 cases are cardiac stresses following hypoxemia, direct effects of own COVID-19 on heart tissue, indirect inflammatory reactions, downregulation of angiotensin-converting enzyme 2 (ACE2) system and sum of these factors $(10,11)$. The other probable mechanism is cytokine storm (a severe immune response in which very large amounts of cytokines are released quickly into the bloodstream within a short period of time) and myocardial damage caused by hypoxemia and respiratory dysfunction (11).

Serological findings of liver in this patient showed hypoalbuminemia and nonsignificant liver function. Histopathological observations showed microvesicular steatosis in hepatocyte associated with inflammatory cells in the periportal area of the liver tissue. Fan et al., in a retrospective study which was conducted on 148 COVID-19 patients, showed that about $37.2 \%$ of liver dysfunctions were associated with longer hospitalization period. They reported that $57 \%$ of their patients developed liver dysfunction after receiving medications (Lopinavir and Ritonavir) (6). Similarly, in the present case, Lopinavir and Ritonavir were prescribed after admission. Therefore, prescribing the mentioned drugs should be done with caution, due to its adverse effects on liver function. Besides, alkaline phosphatase (ALP) increased in this patient; it is considered as a hallmark for epithelial cells in bile ducts. Therefore, it seems that COVID-19 may affect bile ducts epithelium in liver tissue; these changes may be due to the direct effects of the virus on liver tissue or drug side effects. In contrary to our study, a recent study showed no alteration in ALP but elevations in aspartate transaminase (AST) and alanine transaminase (ALT) (12).

Liver damages were shown in SARS and MERS infected patients (12). Human liver tissue can be a good target for COVID-19, because of the high expression of ACE-2, as well as, lung. The ACE- 2 is mainly expressed in cholangiocytes and the expression is low in hepatocytes.

The other finding in our patient was hypoalbuminemia, which is considered as a poor prognostic indicator in ARDS and also acute heart failure (13). The motor symptoms of the patient become worse.

Both ACE2 and Dipeptidyl peptidase-4 are expressed in the central nervous system (CNS). Therefore, the risk of COVID-19 may be elevated in neurological damages such as Alzheimer's and Parkinson's disease. PD is the most common neurodegenerative disorder after Alzheimer and mostly affects elderly people ( $>60$ years). In $\mathrm{PD}$, the dopaminergic neurons are progressively loosed in the Substantia Nigra, both structurally and functionally; it leads to dopamine level reduction in the brain (14). The loss of movement control in PD leads to increased psychological stress gradually. On the other hand, stressful life puts people more susceptible to COVID 19 and respiratory distress; it is too early to decide whether COVID-19 can worsen the disease. This patient had PD for several years. Generally, premature death is higher in PD compared to the normal people. Some determinant factors such as pneumonia, cerebrovascular and cardiovascular diseases affect death occurrence in PD (15). Possibly, in this patient, the COVID-19 had altered the route of dopamine production in CNS, and therefore, it worsened the PD motor symptoms. However, we did not evaluate the patient for PD symptoms specifically (14). The RTqPCR results were negative in all tissues. The clinical and pathological signs of COVID-19 patients in lung, heart and liver are unknown exactly, and further researches are needed.

\section{Conclusion}

The pathological biopsy showed advanced alveolar damage in the lung, peri-portal inflammation in the liver, spread steatosis in the hepatocytes and moderate myocarditis in the heart. However, the RT-qPCR test was negative for the obtained specimens of lung, liver and heart tissues. The alterations in the above-mentioned tissues may be due to the secondary side effects of the COVID-19 or pharmacological treatments.

\section{Acknowledgement}

We would like to express our thanks toward the medical staff of Razi Hospital, especially the general operating room, acute emergency and intensive care unit.

\section{Funding and Ethics}

The informed consent was signed by the patient's family; all procedures were conducted under the 
guidelines of Guilan University of Medical Sciences (Ethic code number: IR.GUMS.REC.1399.012).

\section{Conflict of Interest}

We declare no competing interests.

\section{References}

1. Huang C, Wang Y, Li X,et al. Clinical features of patients infected with 2019 novel coronavirus in Wuhan, China. The lancet 2020;395(10223):497506. [DOI:10.1016/S0140-6736(20)30183-5]

2. Bai Y, Yao L, Wei T,et al. Presumed asymptomatic carrier transmission of COVID-19. JAMA. 2020;323(14):1406-7.

[DOI:10.1001/jama.2020.2565]

3. Long B, Brady WJ, Koyfman A, Gottlieb M. Cardiovascular complications in COVID-19. Am J Emerg Med. 2020. 38(7):1504-1507 [DOI:10.1016/j.ajem.2020.04.048]

4. Lindner D, Fitzek A, Bräuninger $\mathrm{H}$,et al. Association of cardiac infection with SARS-CoV-2 in confirmed COVID-19 autopsy cases. JAMA Cardiol. 2020.5(11):1281-1285 [DOI:10.1001/jamacardio.2020.3551]

5. Chen L, Li X, Chen M, Feng Y, Xiong C. The ACE2 expression in human heart indicates new potential mechanism of heart injury among patients infected with SARS-CoV-2. Cardiovasc Res .2020;116(6):1097-100.

[DOI:10.1093/cvr/cvaa078]

6. Fan Z, Chen L, Li J,et al. Clinical features of COVID-19-related liver damage. Clin Gastroenterol Hepatol. 2020.18(7):1561-1566 [DOI: $10.1101 / 2020.02 .26 .20026971]$

7. Komiya K, Ishii H, Umeki K,et al. Computed tomography findings of aspiration pneumonia in 53 patients. Geriatr Gerontol Int. 2013;13(3):580-5. [DOI:10.1111/j.1447-0594.2012.00940.x]
8. Simpson S, Kay FU, Abbara S,et al. Radiological society of North America expert consensus statement on reporting chest $\mathrm{CT}$ findings related to COVID-19. Endorsed by the Society of Thoracic Radiology, the American College of Radiology, and RSNA. Radiology: Cardiothoracic Imaging 2020;2(2):e200152.

[DOI:10.1148/ryct.2020200152]

9. Li YC, Bai WZ, Hashikawa T. The neuroinvasive potential of SARS-CoV2 may play a role in the respiratory failure of COVID-19 patients. J Med Virol. 2020;92(6):552-5. [DOI:10.1002/jmv.25728]

10. Akhmerov A, Marbán E. COVID-19 and the heart. Circ Res. 2020;126(10):1443-55. [DOI:10.1161/CIRCRESAHA.120.317055]

11. Manolis AS, Manolis TA. Cardiovascular complications of the coronavirus (COVID-19) infection: COVID-19 and the heart. Rhythmos. 2020;15(2):23-8.

12. Chai $\mathrm{X}, \mathrm{Hu} \mathrm{L}$, Zhang $\mathrm{Y}$,et al. Specific ACE2 expression in cholangiocytes may cause liver damage after 2019-nCoV infection. bioRxiv. 2020. [DOI:10.1101/2020.02.03.931766]

13. Bonilla-Palomas JL, Gámez-López AL, MorenoConde M,et al. Hypoalbuminemia in acute heart failure patients: causes and its impact on hospital and long-term mortality. J Cardiac Failure. 2014;20(5):350-8.

[DOI:10.1016/j.cardfail.2014.01.016]

14. Bernstein HG, Dobrowolny H, Keilhoff G, Steiner J. Dipeptidyl peptidase IV, which probably plays important roles in Alzheimer disease (AD) pathology, is upregulated in $\mathrm{AD}$ brain neurons and associates with amyloid plaques. Neurochem Int. 2018;114:55-7. [DOI:10.1016/i.neuint.2018.01.005]

15. Scorza FA, Fiorini AC, Scorza CA, Finsterer J. Cardiac abnormalities in Parkinson's disease and Parkinsonism. J Clin Neurosci. 2018;53:1-5. [DOI:10.1016/j.jocn.2018.04.031]

\section{How to Cite This Article:}

Naseri E, Mohammadghasemi F, Shams F, Saliminasab S, Karimian P, Rahbar Taramsari M, et al Histopathological and Serological Findings of Lung, Liver and Heart in a Patient with Corona Virus Disease2019 (COVID -19): A Case Report. J Adv Med Biomed Res. 2021; 29 (134) :183-188

Download citation:

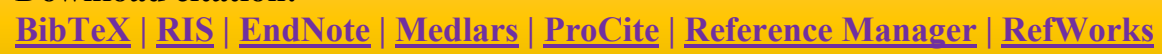

